\title{
Assistência de enfermagem a criança e ao adolescente em situação de violência doméstica
}

\author{
Rosana Alves de Melo ${ }^{1}$; Sinara de Lima Souza ${ }^{2}$; Cristiane Souza Bezerra ${ }^{3}$; \\ Flávia Emília Cavalcante Valença Fernandes ${ }^{4}$
}

Resumo: O Objetivo do presente estudo foi analisar a produção científica nacional e internacional, acerca dos aspectos que envolvem a assistência de enfermagem a crianças e adolescentes em situação de violência doméstica. Métodos: Artigos indexados nas bases de dados: Literatura Latino-Americana em Ciências da Saúde, Medical Literature and Retrieval System Online e Base de Dados em Enfermagem, publicados no período de 2008 a 2014. Resultados: Os enfermeiros não compreendem a violência doméstica em todos os seus aspectos. A falta de conhecimento científico sobre o assunto, a inexistência de protocolos nos serviços que direcionem as condutas profissionais, a desarticulação da equipe multiprofissional e o descrédito na rede de proteção são os principais limites e desafios na prestação da assistência de enfermagem a crianças e adolescentes em situação de violência doméstica. Conclusão: Há necessidade de investir no ensino dentro das instituições formadoras e assistenciais, para favorecer a reflexão dos enfermeiros no desenvolvimento de práticas assistencialistas humanizadas e efetivas no combate à violência doméstica.

Descritores: Violência doméstica; Criança; Adolescente; Enfermagem.

\section{Nursing care for children and adolescents in domestic violence situations}

\begin{abstract}
Abtract: The objective of the present study was to analyze the national and international scientific production on the aspects that involve nursing assistance to children and adolescents in domestic violence situations. Methods: Articles indexed in the databases: Latin American Literature in Health Sciences, Medical Literature and Retrieval System Online and Database of Nursing, published between 2008 and 2014. Results: Nurses do not understand domestic violence in all Its aspects. The lack of scientific knowledge on the subject, the lack of protocols in the services that guide the professional conduct, the disarticulation of the multiprofessional team and the disrepute in the protection network are the main limits and challenges in the provision of nursing assistance to children and adolescents in Situation of domestic violence. Conclusion: There is a need to invest in education inside the educational and welfare institutions, to promote the reflection of nurses in the development of humane and effective welfare practices in combating domestic violence.
\end{abstract}

Keywords: Domestic violence; Child; Adolescent; Nursing.

\footnotetext{
${ }^{1}$ Enfermeira. Mestre em Enfermagem pela Universidade estadual de Feira de Santana/BA. Docente Assistente. Universidade de Pernambuco, Petrolina, Pernambuco, Brasil. E-mail: rosana.melo@upe.br;

${ }^{2}$ Doutora em Ciências. Universidade Estadual de Feira de Santana, Feira de Santana, Bahia, Brasil, E-mail: sinaradd@yahoo.com.br;

${ }^{3}$ Enfermeira. Empresa Brasileira de Serviços Hospitalares. Salvador, Bahia, Brasil. E-mail: crissb_ped@yahoo.com.br;

${ }^{4}$ Enfermeira. Docente Assistente. Universidade de Pernambuco. Petrolina, Pernambuco, Brasil. E-mail: flavia.fernandes@upe.br. Autor correspondente: Rosana Alves de Melo. Rodovia BR 203, Km 2, s/n - Vila Eduardo, Petrolina - PE, 56328-903. Fone: (87) 3866-6468. E-mail: rosana.melo@upe.br.
} 
Id on Line Revista Multidisciplinar e de Psicologia

Id on Line Multidisciplinary and Psycology Journal

\section{Introdução}

A violência é considerada um fenômeno complexo, que manifesta-se de diversas formas, em diferentes contextos, e tem o potencial de causar dano capaz de afetar a saúde individual e coletiva dos envolvidos. Nesse contexto, quando analisa-se os eventos violentos, ver-se que os mesmos provocam diversas consequências, sendo essas capazes de devastar, em maior ou menor grau, a vida de quem a sofre.

Assim, levando em consideração as múltiplas facetas da violência, pode-se observar que, devido a diversos aspectos, as crianças e os adolescentes estão incluídos entre os grupos humanos mais vulneráveis aos eventos violentos e muitas vezes estes ocorrem no contexto familiar, caracterizando-se como um problema de grande relevância social e científica (SILVA; FERRIANI; IOSSI, 2011). Essa particularidade se dá devido ao fato desses grupos encontrarem-se em um processo de crescimento e desenvolvimento mais intenso, e com características peculiares (FERRARI et al., 2008).

A violência que ocorre em casa, é denominada de violência doméstica, e a mesma tem sido objeto de reflexões e pesquisas na área da saúde, por hoje em dia, serem os profissionais dessa área os primeiros a terem contato, na maioria das vezes, com as vítimas diante das sequelas visíveis, e às vezes invisíveis, e por se constituir em um fenômeno que necessita de prevenção, detecção, intervenção e tratamento, de acordo com cada situação.

A violência doméstica contra criança e adolescente pode ser definida como atos ou omissões praticados por pais, parentes ou responsável em relação à criança ou adolescente que sendo capaz de causar à vítima dor ou dano de natureza física, sexual ou psicológica implica, de um lado, uma transgressão do poder de proteção do adulto e, do outro, numa coisificação da infância, negando seus direitos (AZEVEDO; GUERRA, 2011).

Essa violência é considerada uma problemática cultural, pois está fortemente presente nas relações familiares desde a antiguidade, apesar de toda difusão da informação a respeito desse fenômeno, e ocorre em qualquer contexto, independente do nível socioeconômico, e quando praticada contra crianças e adolescentes é conhecida como vitimização ou síndrome dos maus tratos infantis, e pode ser classificada como física, sexual, psicológica e negligência (NIJAINE; ASSIS; CONSTANTINO, 2009; GOMES et al., 2010; LIMA et. a 1., 2011). 
Id on Line Revista Multidisciplinar e de Psicologia

Id on Line Multidisciplinary and Psycology Journal

Por esse fenômeno apresentar uma grande repercussão na vida de crianças e adolescentes, o Estatuto da Criança e do Adolescente foi criado no intuito de assegurar os direitos desses indivíduos e manter sua proteção. O ECA assegura o direito à convivência comunitária e familiar, à livre expressão de opiniões e crenças, o direito de brincar, de praticar esportes e de se divertir. Cabe aos adultos preservar-lhes a integridade física, moral e psíquica, pondo-os a salvo de qualquer tratamento desumano, violento ou constrangedor (BRASIL, 1990; BRASIL, 2008; FALEIROS; FALEIROS, 2008).

Nesse sentido, todos profissionais envolvidos com a assistência em qualquer nível, devem tomar rapidamente as providências necessárias diante de casos suspeitos ou confirmados de violência, para impedir que o problema se agrave, a ponto de causar a morte da vítima ou do agressor. $\mathrm{O}$ enfrentamento da violência requer estratégias bem definidas e que tenham participação da sociedade, dos profissionais e dos gestores públicos, principalmente quando envolve crianças e adolescentes (MARCHEZAN et. al., 2009).

Dentre os profissionais que prestam essa assistência, e necessitam saber abordar as vítimas, está o enfermeiro, que desempenha papel essencial no setor saúde, por possuir uma visão abrangente sobre a atenção nos diversos níveis e ocupar cargos de gerência e intervenção nas políticas públicas. Assim, necessita compreender a violência doméstica contra crianças e adolescentes como um problema abrangente, buscando concentrar esforços para atender os grupos nas suas necessidades e dar resolutividade aos problemas.

Sobre essa questão, enfatiza-se que a atuação da equipe de enfermagem, quando envolve a temática da violência, ainda se dá de forma fragmentada e sem distinção clara das atribuições de cada profissional que compõe a equipe, sendo que deve constituir um processo de atendimento que inclui identificação, intervenção, seguimento, avaliação e encaminhamentos, devendo esse atendimento ser feito de acordo com os princípios do SUS e compreender ações de prevenção, promoção e reabilitação da saúde das vítimas e de suas famílias (SILVA; FERRIANI; IOSSI, 2011).

Diante das colocações aqui elencadas, observa-se que o atendimento às crianças e adolescentes em situação de violência doméstica tem sido um desafio cada vez maior para os profissionais de Enfermagem, por dificuldades decorrentes de sua formação profissional e também pela dificuldade cultural de compreender que diversas formas de violência que podem ocorrer dentro do lar, local que deveria ser primordialmente repleto de acolhimento e proteção. 
Id on Line Revista Multidisciplinar e de Psicologia

Id on Line Multidisciplinary and Psycology Journal

Nesta perspectiva, levando em consideração os aspectos que envolvem crianças e adolescentes em situação de violência doméstica, bem como a importância de um atendimento de enfermagem que contemple as necessidades sociais e de saúde desses indivíduos, esse estudo objetivou analisar a produção científica nacional e internacional, acerca dos aspectos que envolvem a assistência de enfermagem a crianças e adolescentes em situação de violência doméstica.

Considera-se que a evidência desta assiduidade às publicações sobre a temática, pode oferecer melhor suporte para a inovação da prática profissional, através da melhoria do ensino na graduação. Dessa forma, foi elaborada a seguinte questão norteadora: quais informações podem ser encontradas nas publicações cientificas nacionais e internacionais acerca da assistência de enfermagem a criança e adolescente em situação de violência doméstica?

\section{Método}

Realizou-se uma Revisão Integrativa, com coleta de dados realizada a partir de fontes secundárias, por meio de levantamento bibliográfico no período de 2008 a 2014. A Revisão Integrativa da Literatura é um método que tem a finalidade de proporcionar a síntese de conhecimento e a incorporação da aplicabilidade de resultados de estudos significativos na prática, que dão suporte para a tomada de decisão e a melhoria da prática clínica, além de apontar lacunas do conhecimento que precisam ser preenchidas com a realização de novos estudos (SOUZA; SILVA; CARVALHO, 2010).

A pesquisa bibliográfica é uma das melhores formas de iniciar um estudo, buscando-se semelhanças e diferenças entre os artigos levantados nos documentos de referência. A compilação de informações em meios eletrônicos é um grande avanço para os pesquisadores, democratizando o acesso e proporcionando atualização frequente (SOUZA; SILVA; CARVALHO, 2010). Dessa forma, para o levantamento dos artigos na literatura, realizou-se uma busca através da Biblioteca Virtual em Saúde (BVS), nas seguintes bases de dados: Literatura Latino-Americana em Ciências da Saúde (LILACS), Medical Literature Analysis and Retrieval System Online (Medline) e a Base de Dados em Enfermagem (BDENF). 
Id on Line Revista Multidisciplinar e de Psicologia

Id on Line Multidisciplinary and Psycology Journal

A coleta de dados ocorreu em janeiro de 2015, onde foi empregado os Descritores em Ciências da Saúde (DeCS) Violência Doméstica; Criança; Adolescentes; Enfermagem, resultando, incialmente em um total de 74 artigos originais. Foram estabelecidos como critérios de inclusão: I - artigos originais (pesquisa ou revisão); II - artigos publicados em português, inglês ou espanhol; III - resumos com objetivos, métodos, e resultados claramente definidos no texto; IV - artigos cujo resultados abordassem os aspectos que envolvem a assistência de enfermagem a crianças e adolescentes em situação de violência doméstica.

Ao ser estabelecidos os critérios de inclusão, realizou-se uma filtragem das 74 publicações encontradas inicialmente, resultando em 29 artigos para a revisão, tomando como base os resumos. Após a leitura dos resumos, foram excluídos 17 artigos que não preencheram os critérios de inclusão. Dessa maneira, restaram 12 artigos incluídos nessa revisão, analisados na íntegra, contemplados nessa Revisão Integrativa da Literatura. Foi feita a leitura exploratória seletiva, analítica e interpretativa desses artigos, para que fosse iniciada a discussão dos dados encontrados.

\section{Resultados e Discussão}

A base de dados que continha o maior número de publicações e que foram selecionados foi a LILACS (08), seguida pela BDENF (03) e a MEDLINE (01).

Diante da leitura feita e com base na questão norteadora, puderam ser elaboradas três categorias, para melhor entendimento dos resultados:

\section{Compreensão do profissional enfermeiro sobre violência doméstica contra criança e adolescente}

O ambiente familiar é considerado uma referência na proteção de crianças e adolescentes, quando considera-se que a família, pelos laços afetivos estabelecidos, tem o dever de proteger e assegurar o bem estar uns dos outros. A criança e o adolescente, pelo processo de crescimento e desenvolvimento mais acentuado, tem nesse ambiente um lócus social de extrema 
Id on Line Revista Multidisciplinar e de Psicologia

Id on Line Multidisciplinary and Psycology Journal

importância para seu desenvolvimento biopsicossocial, onde a família favorece a formação da personalidade desses indivíduos.

Ambiente familiar é uma expressão que geralmente designa acolhimento, aconchego, cuidado, conforto, confiança e, acima de tudo, proteção. Entretanto, para muitas crianças, essa não é uma realidade, pois, o ambiente é sinônimo de sofrimento, medo e maus- tratos (PAIXÃO et al., 2013). Nesse sentido, destaca-se que diante dos diversos tipos de violência existentes, a violência doméstica contra crianças e adolescentes está tomando cada vez mais proporções alarmantes e os maiores agressores são os próprios membros da família, e um dos maiores desafios para os profissionais de saúde, é reconhecer e intervir de forma integral nos diversos casos de violência que tem contato na sua rotina profissional.

A violência doméstica contra crianças e adolescentes pode assumir características de agressão física, sexual, negligência e psicológica e, por essa razão, deve ser denunciada. Assim, cabe ao profissional de saúde saber como este tipo de violência ocorre, suas formas, e estar apto a reconhecer os casos que vivenciar, se estes caracterizam situações em que esses indivíduos foram ou estão sofrendo alguma forma de agressão, considerando os vários tipos especificados (BARBOSA; PEGORARO, 2008).

Percebe-se que na compreensão do que seja violência doméstica contra criança e adolescente, não existe uma denominação concreta nos estudos analisados, sendo de início ambígua, onde os conceitos descritos estão mais baseados no senso comum, do que em aspectos científicos relacionados ao fenômeno (THOMAZINE; OLIVEIRA; VIEIRA, 2009; DIAS et. al., 2013; ZANELATTO et. al., 2012). Mesmo que sejam retratados alguns aspectos que envolvem a violência doméstica, nenhum deles traz de fato, nos relatos dos participantes, a compreensão de como se dá, em que ambiente e os seus tipos.

Alguns estudos, ao denominarem a violência doméstica contra criança e adolescente, trouxeram que essa se dá através de abusos físicos e psicológicos, sendo que até foram descritas ações que englobam a negligência, porém, essa não sendo reconhecida como violência (THOMAZINE; OLIVEIRA; VIEIRA, 2009; ZANELATTO et. al., 2012). Ademais, outros estudos abordam nas suas denominações que a violência doméstica contra criança e adolescente está intimamente relacionada a omissão do cuidado prestado, que envolve abandono e negligência do cuidado, o que pode levar a danos físicos e emocionais irreversíveis (SILVA et. al., 2009; ABRANCHES; ASSIS, 2011). 
Id on Line Revista Multidisciplinar e de Psicologia

Id on Line Multidisciplinary and Psycology Journal

Ressalta-se que a negligência, enquanto violência, ainda é pouco perceptível em alguns cenários, pois muitas situações que ocorrem nos lares ainda são vistas como naturais e que não necessitam de intervenção. Outras, são erroneamente julgadas como violência. Dessa forma, é importante analisar a complexidade dessas situações, pelo fato de que o limite entre o que podemos reconhecer como negligência do cuidado dos pais e responsáveis para com os filhos e o julgamento moral dos profissionais em relação as situações familiares baseadas num modelo de cuidado alheio ao contexto sociocultural das famílias, é limitado, e isso pode levar a um diagnóstico errôneo (ABRANCHES; ASSIS, 2011).

A violência sexual, mesmo tendo um potencial de deixar sequelas irreparáveis, foi citada em poucos estudos, como um tipo de violência doméstica contra criança e adolescente. Isso pode estar associado ao fato de ser um tipo de violência subjetiva, que deixa poucas marcas aparentes, na maioria das situações o que faz com que alguns profissionais a percebam enquanto violência física, ou ainda se deva a existência de tabus em relação esse tipo de abuso (THOMAZINE; OLIVEIRA; VIEIRA, 2009; ZANELATTO et. al., 2012; SILVA et. al., 2009).

A violência doméstica é vista tanto pela família, quanto pelos profissionais, como uma expressão cultural de educar que tende a se perpetuar de geração para geração. Nesse caso, em muitas situações é aceita de forma natural e serve de medida de educativa, porém o que diferencia uma situação de outra é a intensidade das agressões (ZANELATTO et. al., 2012; PAIXÃO et. al., 2013; DIAS et. al., 2013). No entanto, sabe-se que toda ação que causa dor física numa criança ou adolescente, desde um simples tapa, até um espancamento fatal representa um ato contínuo de violência, e que a imposição de limites e disciplina dos filhos pode ser efetiva, através de outras formas mais eficazes de educação (JUCÁ, 2010).

A violência doméstica contra crianças e adolescentes, por muitas vezes é vista como um ciclo que decorre de experiências agressivas sofridas pelos pais no passado, dentro do seu convívio familiar, fazendo-o naturalizar e repetir um comportamento violento nas sua relação com os demais membros da família, principalmente os filhos (ABRANCHES; ASSIS, 2011). Outra particularidade encontrada nos estudos avaliados, é que as questões sociais, como desemprego, baixa escolaridade, falta de planejamento familiar, pobreza, entre outros, são considerados motivos que concorrem para a ocorrência da violência contra crianças e adolescentes (THOMAZINE; OLIVEIRA; VIEIRA, 2009; SILVA et. al., 2009). 
Id on Line Revista Multidisciplinar e de Psicologia

Id on Line Multidisciplinary and Psycology Journal

As questões sociais estão intimamente relacionadas a ocorrência de atos violentos no ambiente doméstico, e nesse aspecto, o fato de os adultos terem sofrido violência na infância e juventude favorecerá a sua perpetuação. Assim, a violência se constitui como um fenômeno sociocultural relacionada intimamente às particularidades dos grupos sociais, ao modo dos indivíduos compreenderem (coletivamente) as situações vividas, ao estabelecimento de regras e condutas, entre outros. Dessa maneira, pode ser considerada ou não como um ato violento dependendo da cultura e da sociedade que o indivíduo está inserido, e cada um tem uma maneira individual de vivenciar tudo isso (PIERANTONI; CABRAL, 2009; CHUNG et. al, 2009).

Diante dos estudos avaliados, percebe-se que os profissionais até compreendem a violência doméstica contra crianças e adolescentes, porém ainda não a concebem de forma integral, levando em consideração todo o seu contexto, seus tipos e os aspectos que a envolvem. Assim, o profissional, além de precisar saber conceituar esse fenômeno de uma forma linear, necessita fazer uma avaliação das situações que tiver contato, e pensar a violência no âmbito de relações, dentro de um contexto, que pode ser consequência de uma dinâmica relacional complexa e que envolve muitos sujeitos.

\section{Despreparo teórico-prático da enfermagem na assistência à criança e adolescente em situação de violência doméstica}

Dados epidemiológicos comprovam que as crianças e adolescentes são os grupos populacionais mais vulneráveis a sofrer algum tipo de violência, através da violação de seus direitos, o que tem o potencial de causar danos à saúde física e emocional desses indivíduos. E esse fenômeno está cada vez mais presente na vida cotidiana da população, trazendo graves consequências para a qualidade de vida não apenas de crianças e adolescentes, mas de toda coletividade (MONTEIRO et. al., 2009).

A violência doméstica é um fenômeno complexo e que necessita de medidas mais que pontuais para que possa ser combatida e/ou evitada. É um dos problemas que exige reestruturação das capacidades dos profissionais de saúde que prestam assistência aos indivíduos, principalmente os enfermeiros, por serem componentes chave nas equipes de saúde. 
Id on Line Revista Multidisciplinar e de Psicologia

Id on Line Multidisciplinary and Psycology Journal

E nesse aspecto, precisam estar aptos para detectar e decodificar os sinais e sintomas entre crianças e adolescentes em situação de violência (SILVA et. at., 2009; PESCE, 2009).

Porém, dos estudos analisados, foi quase unânime que os enfermeiros, tanto da atenção básica, como da assistência hospitalar, tem dificuldades em identificar e conduzir os casos de violência que tem contato, principalmente quando as vítimas são crianças ou adolescentes. $\mathrm{E}$ nesse sentido, também demonstram desconhecer quais as principais condutas assistencialistas e os encaminhamentos que obrigatoriamente devem ser dados aos casos (PAIXÃO et. al., 2013; THOMAZINE; OLIVEIRA; VIEIRA, 2009; ZANELATTO ET AL., 2012; DIAS et. al., 2013; WOISKI; ROCHA, 2010; ARAGÃO et. al., 2013).

Essa conduta é justificada, na maioria das vezes, pela dificuldade em entender a linguagem da criança ou do adolescentes, que tem uma forma peculiar de se expressar, seja verbal ou comportamental, principalmente quando violentadas (ZANELATTO et. al., 2012); pelo fato de ainda manterem a visão biomédica de se preocuparem em tratar somente as lesões visíveis e aquilo que for mais subjetivo, passa despercebido (THOMAZINE; OLIVEIRA; VIEIRA, 2009); ou mesmo a insegurança teórica sobre a temática, que provém de uma formação acadêmica deficiente e que restringe o profissional a prestar uma assistência limitada e pontual (DIAS et. al., 2013; WOISKI; ROCHA, 2010).

Outra dificuldade que foi encontrada nas publicações que abordam a temática é que muitos enfermeiros só realizam a notificação, e em seguida acreditam que a melhor conduta é encaminhar os casos para que os demais profissionais da equipe, como o psicólogo e o assistente social, possam resolver, não havendo discussão dos casos entre os demais membros ou serviços, nem tampouco articulação das condutas realizadas (DIAS et. al., 2013; SILVA et. al., 2009; ARAGÃO et. al., 2013). Esse comportamento pode estar associado ao fato de não saber como lidar com o problema ou mesmo por acreditar que não se trata de algo que deve ser resolvido pela enfermagem, e sim pelos demais profissionais assistentes.

Sabem-se que todos os profissionais da equipe de saúde precisam estar capacitados e se reconhecerem como responsáveis pela condução dos casos suspeitos e confirmados de violência, onde a articulação entre os serviços e o trabalho em equipe devem prevalecer, com o propósito de romper esse ciclo aos vitimizados e seus familiares (SILVA et. al., 2009). O trabalho articulado favorece a abordagem, a investigação, o diagnóstico, atendimento e 
Id on Line Revista Multidisciplinar e de Psicologia

Id on Line Multidisciplinary and Psycology Journal

encaminhamentos devidos, da melhor forma possível e mantem a qualidade da assistência prestada.

\section{Limites e desafios na assistência de enfermagem a criança e adolescente em situação de violência doméstica}

Com relação aos limites e desafios registrados pelos enfermeiros no atendimento em criança e adolescente em situação de violência doméstica, está a inexistência de protocolos que normatizem os cuidados e encaminhamentos que devem ser prestados, a falta de articulação que existe entre os membros da equipe de saúde, onde cada uma age por si só, e também o despreparo emocional do profissional em lidar com situações vulneráveis e que leva o mesmo a adquirir postura inadequada frente ao fenômeno (ABRANCHES; ASSIS, 2011; THOMAZINE; OLIVEIRA; VIEIRA, 2009; SALCEDO ET. AL., 2011; DIAS et. al., 2013).

Ressalta-se que um serviço de saúde que não esteja organizado e que não oferece condições mínimas satisfatórias para que o profissional desenvolva seu trabalho da melhor forma, gera sentimentos de impotência e frustração, assim como pressupõe um trabalho desarticulado, onde os membros da equipe não dialogam e nem tampouco sejam parceiros na situações complexas de atendimentos, podem exacerbar esses sentimentos negativos e desfavorecer a resolubilidade dos casos. E outra ressalva, é que os serviços precisam direcionar o olhar também às necessidades dos trabalhadores que prestam assistência, sabendo que o indivíduo que cuida de quem sofre violência também precisa de cuidado (CARVALHO, 2012).

A falta de credibilidade na rede de proteção a violência também foi mencionada nos estudos analisados, como um limite em se proceder com os devidos encaminhamentos que são preconizados, sendo alegado a falta de infraestrutura adequada dos serviços de referência e a não resolubilidade dos casos encaminhados (THOMAZINE; OLIVEIRA; VIEIRA, 2009; SALCEDO et. al., 2011; DIAS et. al., 2013). Nesse sentido, a intersetorialidade com outros serviços, como o Conselho Tutelar, deve ser bem estruturada e organizada, e que todos saibam suas responsabilidades e funções, para que os encaminhamentos sejam resolutivos e possam minimizar ou combater os casos de violência existentes. 
Id on Line Revista Multidisciplinar e de Psicologia

Id on Line Multidisciplinary and Psycology Journal

Das publicações citadas nesta revisão, mais da metade traz como desafio e limite na assistência de enfermagem a criança e adolescente em situação de violência doméstica o despreparo técnico-científico sobre a temática, mostrando que os profissionais não recebem as orientações necessárias durante a formação acadêmica; que os conteúdos são insuficientes; e que há uma desarticulação entre teoria e prática. E também referem não ter participado de nenhuma capacitação sobre violência nos atuais serviços de saúde onde atuam. Assim, não se sentem devidamente preparados para atuarem nessas situações (THOMAZINE; OLIVEIRA; VIEIRA, 2009; SILVA et. al., 2009; SALCEDO ET AL., 2011; DIAS et. al., 2013; PAIXÃO ET. AL., 2013; ARAGÃO et. al., 2013).

De acordo com essas informações, é importante levar em consideração que a capacitação dos profissionais de saúde é crucial para que possam ser capazes de identificar e diagnosticar os diferentes tipos de violência, a sua presença ou a suspeita nos diferentes casos atendidos, que geralmente oscila entre os aspectos visíveis e invisíveis do fenômeno, e perceber que a assistência envolve não somente ter conhecimento científico, mas conseguir, dentro do contexto existente, articular a teoria com o desempenho integralizado e socializador que o problema exige para que tenha resolutividade (COSTA et. al., 2013; DIAS et. al., 2013).

Um dos estudos analisados buscou identificar os limites e potencialidades de uma ferramenta sistematizadora do cuidado que possibilita visibilizar a violência infantil durante as consultas de enfermagem, e mostrou que mesmo descritos na nomenclatura, os enfermeiros não trouxeram os diagnósticos específicos da violência; as intervenções propostas incluíram cuidados direcionados somente às lesões; não foram sugeridas intervenções que incluíssem a criança ou a família na rede de proteção; e os próprios enfermeiros não se posicionaram como atores sociais para enfrentar as essas necessidades desses indivíduos (APOSTÓLICO; HINO; EGRY, 2013).

Assim, mesmo sabendo que a consulta de enfermagem, quando bem realizada, representa uma importante ferramenta para detecção de casos de violência, não tem o potencial de modificar a realidade da população se não articular ações e serviços que correspondam às expectativas e necessidades dos indivíduos, pois o conjunto de instrumentos e ações é que poderão favorecer uma assistência de qualidade e transformadora da realidade (APOSTÓLICO; HINO; EGRY, 2013). 
Id on Line Revista Multidisciplinar e de Psicologia

Id on Line Multidisciplinary and Psycology Journal

O enfrentamento às situações de violência doméstica, principalmente quando envolve crianças e adolescentes, necessita de uma abordagem intersetorial e multidisciplinar, que pressupõe em sua construção uma micropolítica própria, com a participação de outros atores e saberes na composição do cuidado, no sentido de favorecer uma assistência de qualidade e resolutiva. Nesse contexto, é preciso considerar que há distintas visões sobre as famílias, expectativas e compreensões diferentes sobre o atendimento dos casos, e que a articulação de toda a equipe de saúde promoverá uma assistência efetiva e que favoreça a quebra do ciclo de violência doméstica (MOREIRA et. al., 2014).

\section{Conclusão}

Os estudos trazidos nessa revisão proporcionou subsídios para que se pudesse dimensionar e compreender a totalidade das expressões da violência na vida e saúde desses indivíduos, mas sabendo que muito ainda precisa ser pesquisado no que se refere a essa problemática, no sentido de se buscar um embasamento científico que dê sustentação a práticas de saúde mais efetivas e integralizadas.

Observou-se que mesmo tendo um papel importante na equipe de saúde, o enfermeiro ainda não consegue conceituar os aspectos da violência doméstica contra crianças e adolescentes, e a partir daí, não se reconhece como protagonista no enfrentamento desse problema. Na maioria das vezes isso se deve ao fato de esse profissional não possui conhecimento científico suficiente que possa embasar suas ações diante dos casos, decorrente de uma formação acadêmica deficiente e falta de capacitações nos serviços de saúde.

Percebeu-se que a dificuldade enfrentada pelos enfermeiros para acolher e atender crianças vítimas de violência doméstica nos diversos contextos, decorre não apenas da má formação profissional, mas também da vivência da violência doméstica contra crianças e adolescentes no ambiente de trabalho sem apoio de serviços de referência bem estruturados; a falta de organização/protocolos dos serviços de saúde que assistem os indivíduos; e também a desarticulação entre os próprios membros da equipe de saúde que compõem o serviço. 
Id on Line Revista Multidisciplinar e de Psicologia

Id on Line Multidisciplinary and Psycology Journal

Diante desses entraves, é fundamental que as instituições e ensino e os serviços de saúde se preocupem em incluir, no processo de formação e capacitação desses profissionais, conteúdos que lhes permitam mais assertividade diante do problema da violência doméstica, no sentido de proporcionar uma reorganização das práticas assistencialistas que devem ser focadas nas necessidades dos indivíduos e de suas famílias, visando a prevenção e combate da violência. As escolas, enquanto instituições formadoras, devem oferecer estratégias de prevenção, reconhecimento e combate da violência entre os estudantes, como forma de quebrar esse ciclo.

Para que haja um atendimento eficiente e eficaz por parte dos profissionais envolvidos na atenção da criança/adolescente em situação de violência doméstica que adentrem os serviços de saúde, faz-se necessário um compromisso das instituições da rede e que haja a implementação de protocolos de atendimento que definam claramente o papel de cada membro, instituição, órgão, setores da sociedade e profissionais no atendimento e prevenção da violência, para que assim se construa uma rede hierarquizada, articulada e contínua de ações.

\section{Referências}

ARAGÃO, A.S., ET AL. Abordagem dos casos de violência à criança pela enfermagem na atenção básica. Rev. Latino-Am. Enferm. Jan.-fev. 2013.

ABRANCHES C.D., ASSIS S.G. A (in)visibilidade da violência psicológica na infância e adolescência no contexto familiar. Caderno de Saúde Pública, Rio de Janeiro, p. 843-854, mai. 2011.

AZEVEDO, M.A.; GUERRA, V.N.A. (orgs.) (1993). Infância e violência doméstica: fronteiras do conhecimento. São Paulo: Cortez. $6^{\text {a }}$ ed., 2011.

APOSTÓLICO, M.A.; HINO, P.; EGRY, E.Y. Possibilities for addressing child abuse in systematized nursing consultations. Rev. Esc. Enferm. USP. Vol. 47, n.2, São Paulo, abr. 2013.

BARBOSA, P.Z.; PEGORARO, R.F. Violência doméstica e psicologia hospitalar: possibilidades de atuação diante da mãe que agride. Rev. Saúde Soc. 2008;17(3):77-89.

BRASIL. Estatuto da Criança e do Adolescente (ECA). Lei 8.069 de 13 de julho de 1990. Dispõe sobre o Estatuto da Criança e do Adolescente e dá outras providências. 1990.

BRASIL. Secretaria de Estado de Saúde do Distrito Federal. Manual para Atendimento às Vítimas de Violência na Rede de Saúde Pública do Distrito Federal. In.: Vilela, LF (coord.) - Brasília: Secretaria de Estado de Saúde do Distrito Federal, 2008. 
Id on Line Revista Multidisciplinar e de Psicologia

Id on Line Multidisciplinary and Psycology Journal

CARVALHO, S.D. O enfermeiro e o cuidar multidisciplinar na saúde da criança e do adolescente. São Paulo: Editora Atheneu, 2012.

COSTA, M.C.O.; ET AL. Violência e vitimização na infância e adolescência: a inclusão da escola na reconhecimento e prevenção. Feira de Santana: UEFS Editora, 2013. 80 p.:il.

CHUNG, E.K.; ET AL. Parenting attitudes and infant spanking: the influence of childhood experiences. Pediatrics. 2009; 124(2):278-86.

DIAS, E.P.; ET AL. Enfermeiros no Atendimento de Casos de Violência Doméstica Infantil em Unidades Básicas de Saúde. Rev. Levs/Unesp-Marília, 12 ed., 2013.

FALEIROS, V.P.; FALEIROS, E.S. Ministério da Educação. Escola Que Protege: Enfrentando a violência contra crianças e adolescentes. Brasília: Secretaria de Educação Continuada, Alfabetização e Diversidade, 2 ed., 2008.

FERRARI, D.C.A., ET AL. Reconstrução de vidas: como prevenir e enfrentar a violência doméstica, o abuso e a exploração sexual de crianças e adolescentes. São Paulo: SMADS. SEDES Sapientae, 2008. 96 p.

GOMES, A.V.O., ET AL. A criança vítima de violência doméstica: limites e desafios para a prática de enfermagem. Rev. de Pesq.: cuidado é fundamental [online] 2010. abr/jun. 2(2):902-912.

JUCÁ, M. Palmada pedagógica. Rev. Psiq Cienc \& Saúde, ano V, edição 57, set., 2010, p. 3943.

LIMA, M.C.C.S. ET AL. Atenção profisssional na atenção básica de saúde face à identificação e a notificação da violência infanto-juvenil. Revista Baiana de Saúde Pública. Salvador, p. 118-137, jan-jun. 2011.

MARCHEZAN, S.; ET AL. A enfermagem e a criança vítima de violência sob o olhar de Paterson \&Zderad. CogitareEnferm, 2009, jan/mar; 14(1):44-51.

MOREIRA, T.N.F., ET AL. A construção do cuidado: o atendimento às situações de violência doméstica por equipes de saúde da família. Rev. Saúde \& Soc. Vol. 23, n. 3, São Paulo Jul/Sep. 2014.

MONTEIRO, E.M.L.M.; ET AL. Violência contra crianças e adolescentes: rompendo o silêncio. Rev. Rene. Fortaleza, v. 10, no 3, p. 107-116. Jul/set 2009

NIJAINE, K.; ASSIS, S.G.; CONSTANTINO, P. Curso Impactos da violência na saúde. In: Cavalcanti, FG; Schenker M. Violência, família e sociedade. Rio de Janeiro: Fundação Oswaldo Cruz - Fiocruz/EAD; 2009. p. 58-72. 
Id on Line Revista Multidisciplinar e de Psicologia

Id on Line Multidisciplinary and Psycology Journal

PAIXÃO, G.P.N., ET AL. Violência intrafamiliar contra criança: atribuições do profissional de enfermagem. Rev. Eletr. Fainor, Vitória da Conquista, v.6, n.2, jul./dez. 2013. p.22-39.

PIERANTONI, L.M.M.; CABRAL, I.E. Crianças em situação de violência de um ambulatório do Rio de Janeiro: conhecendo seu perfil. Esc. Anna Nery. Rev. Enferm. 2009; 13(4):699-707.

PESCE, R. Violência familiar e comportamento agressivo e transgressor na infância: uma revisão da literatura. Rev. Cienc. Saúde Colet. 2009;14(2):507-18.

SALCEDO, B.D.M., ET AL. Violência doméstica e enfermagem: da percepção do fenômeno à realidade cotidiana. Avances en Enfermería. Vol. 29, n. 2. Jul-dez, 2011

SILVA, P.A.; ET AL. Notificação da violência intrafamiliar contra crianças e adolescentes na percepção dos profissionais de saúde. Rev. Ciências Cuidado e Saúde. v. 8, n. 1, jan.-mar., 2009. p. 56-62.

SILVA, L.M.P.; FERRIANI, M.G.C.; IOSSI, M.A. Atuação da enfermagem frente à violência sexual contra crianças e adolescentes. Rev. Bras. Enferm. V. 64, n. 5, Brasília, set/oct, 2011.

SOUZA, M.T.; SILVA, M.D.; CARVALHO, R. Revisão integrativa: o que é e como fazer. Rev. Einstein. 2010; 8(1):102-6.

THOMAZINE, A.M.; OLIVEIRA, B.R.G.; VIEIRA, C.S. Atenção a crianças e adolescentes vítimas de violência intrafamiliar por enfermeiros em serviços de pronto-atendimento. Rev. Eletr. Enf. [online]. 2009;11(4):830-40.

WOISKI, R.O.S.; ROCHA, D.L.B. Cuidado de enfermagem à criança vítima de violência sexual atendida em unidade de emergência hospitalar. Esc Anna Nery. Rev Enferm. Jan-mar, 2010; 14 (1): 143-50.

ZANELATTO, P.F. ET AL. Violência contra crianças e adolescentes: significados e atitudes por equipes da estratégia saúde da família. Rev. Ciencia y Enfermería XVIII, 2012. (2): 4149.

Como citar este artigo (Formato ABNT):

MELO, S.A.; SOUZA, S.L.; BEZERRA, C.S.; FERNANDES, F.E.C.V. Assistência de enfermagem a criança e ao adolescente em situação de violência doméstica. Id on Line Revista Multidisciplinar e de Psicologia, NovDez. de 2016, vol.10, n.32, p. 245-259. ISSN: 1981-1179.

Recebido: 16/11/2016

Aceito: $21 / 11 / 2016$ 